\title{
-NOTES-
}

\section{EFFECTS OF GRAVITATIONAL OR ELECTROMAGNETIC FIELDS ON FLUID MOTION*}

\author{
Bx CHIA-SHUN YIH (University of Michigan)
}

Summary. It is shown in this paper that the effect of gravity on a stratified fluid is to inhibit steady motion in the direction of gravity; that, for small values of the magnetic viscosity, the effect of a main magnetic field is to make steady weak motions of a fluid independent of the distance along the lines of force; and that, again for small values of the magnetic viscosity, the effect of a uniform electric field is to make steady weak motions of a fluid rotationally symmetric with respect to an axis in the direction of the field. These results, together with a similar one of Proudman (1916) [1] for a fluid with general rotation, enable one to state that the effects of rotation, gravity, and electromagnetic fields (for small magnetic viscosity) are to endow the fluid with a certain anisotropic rigidity by "stiffening" it along the vorticity lines, the isopycnic surfaces or lines, or the lines of force, as the case may be. In the case of a weak steady motion or relative motion, this "stiffening" has the effect of reducing the a priori number of physical or at least mathematical dimensions of the motion by one.

1. Introduction. Since Proudman [1] showed that the effect of rotation on weak steady relative motion of an inviscid fluid is to make it two-dimensional, in the sense that the motion is independent of the distance along the axis of rotation, experiments performed by Taylor [2], Long [3], and others have largely supported his assertion. Recently, Gariel's experiments with a stratified fluid and Lehnert's with mercury have indicated similar effects of gravity and of a magnetic field, respectively. The purpose of this paper is to describe these effects, to predict the effect of an electric current on weak steady motions of a fluid, to explain all these effects mathematically and, as far as possible, to bring out their similarity to one another. The fluid is assumed to be inviscid and incompressible throughout and, in the case of an electromagnetic field, to have negligible magnetic viscosity.

2. Effect of rotation. For convenience of comparison and for completeness, Proudman's demonstration [1] of the effect of rotation will be briefly presented. If the general rotation of the fluid is $\Omega$, the axis of rotation can be chosen to be the $x$-axis. Two other axes, both rotating with angular velocity $\Omega$, can be chosen to provide a rotating cartesian frame of reference, with coordinates $(x, y, z)$. The velocity components relative to this rotating frame in the directions of $x, y$ and $z$ will be denoted by $u, v$, and $w$ respectively. The equations of motion are then (Morgan [4]):

$$
\begin{aligned}
\frac{D u}{D t} & =-\frac{\partial P}{\partial x}, \\
\frac{D v}{D t}-2 \Omega w & =-\frac{\partial P}{\partial y}, \\
\frac{D w}{D t}+2 \Omega v & =-\frac{\partial P}{\partial z},
\end{aligned}
$$

*Received August 2, 1957. 
in which

$$
\frac{D}{D t}=\frac{\partial}{\partial t}+u \frac{\partial}{\partial x}+v \frac{\partial}{\partial y}+w \frac{\partial}{\partial z}
$$

and

$$
P=\frac{p}{\rho}-\frac{1}{2} \Omega^{2}\left(y^{2}+z^{2}\right)+\phi
$$

with $p, \rho$, and $\phi$ as the pressure, density, and the body-force potential, respectively. The equation of continuity is

$$
\frac{\partial u}{\partial x}+\frac{\partial v}{\partial y}+\frac{\partial w}{\partial z}=0
$$

For steady and weak relative motion, Eqs. (1) to (3) become

$$
\begin{aligned}
& \frac{\partial P}{\partial x}=0, \\
& \frac{\partial P}{\partial y}=2 \Omega w, \\
& \frac{\partial P}{\partial z}=-2 \Omega v .
\end{aligned}
$$

By cross-differentiation of Eqs. (7) and (8), it can be readily shown that

$$
\frac{\partial v}{\partial y}+\frac{\partial w}{\partial z}=0
$$

It follows from Eq. (5) that

$$
\frac{\partial u}{\partial x}=0 .
$$

Furthermore, by differentiation of Eqs. (7) and (8) with respect to $x$ it can readily be seen with the aid of Eq. (6) that

$$
\frac{\partial v}{\partial x}=0, \quad \frac{\partial w}{\partial x}=0 .
$$

Equations (10) and (11) indicate that the motion is independent of $x$. Hence, the steady and weak relative motion is two-dimensional. In a loose way, one might say that the general rotation has endowed the fluid with a certain rigidity along the vorticity lines.

It can be readily shown that for symmetric motion the radial velocity component vanishes (so that the streamlines are spirals wound around circular cylinders). This situation can be directly compared with the vanishing of the vertical velocity component in the steady weak motion of a stratified fluid to be treated in the following section, for in the case of rotation one may imagine a virtual gravity acting in the radial direction, according to Mach and Einstein.

3. Effect of gravity. One now turns to the effect of gravity on steady and weak motions of a stratified fluid. Let the $z$-axis be vertical. The equations of motion are 


$$
\begin{aligned}
& \rho \frac{D u}{D t}=-\frac{\partial p}{\partial x} \\
& \rho \frac{D v}{D t}=-\frac{\partial p}{\partial y} \\
& \rho \frac{D w}{D t}=-\frac{\partial p}{\partial z}-g \rho
\end{aligned}
$$

in which $D / D t$, as usual, stands for the substantial differentiation. The equation of incompressibility is

$$
\frac{D \rho}{D t}=0
$$

which permits the equation of continuity to be given by Eq. (5).

The density can be expressed as the sum of a prevailing density $\rho_{1}$, which depends only on $z$, and a perturbation density $\rho^{\prime}$ :

$$
\rho=\rho_{1}(z)+\rho^{\prime} .
$$

For steady weak motion, Eqs. (12) and (13) become

$$
\frac{\partial p}{\partial x}=0, \quad \frac{\partial p}{\partial y}=0
$$

and hence, from Eqs. (14) and (16) one obtains

$$
\frac{\partial \rho^{\prime}}{\partial x}=0, \quad \frac{\partial \rho^{\prime}}{\partial y}=0 .
$$

Equations (17) and (18) are correct to the first order. But from Eqs. (15) and (16) it follows that

$$
u \frac{\partial \rho^{\prime}}{\partial x}+v \frac{\partial \rho^{\prime}}{\partial y}+w \frac{d \rho_{1}}{d z}+w \frac{\partial \rho^{\prime}}{\partial z}=0
$$

so that, from Eqs. (18),

$$
w=0
$$

to the second order. Thus gravity inhibits steady velocity in the vertical direction, and if the disturbance is not strong enough, steady motion can occur in horizontal planes only. This situation is directly comparable to that for symmetric relative motion with general rotation, provided that that motion is steady and weak, as mentioned in the last section. In the present case, it is the isopycnic surfaces or lines that are "stiffened."

Another result comparable to Proudman's can also be obtained. If two-dimensional motion is considered to start with, so that $v$ is zero, Eqs. (5) and (20) yield

$$
\frac{\partial u}{\partial x}=0
$$

Thus the motion will be one-dimensional, in the sense that the state of affairs does not change with $x$-in much the same way that Proudman's motion is independent of $x$. In both cases $x$ is measured in a direction normal to that of gravitation or virtual gravi- 
tation created by rotation. The essential one-dimensional character of steady weak motions of a stratified fluid has been experimentally demonstrated by Gariel [5].

4. Effect of a magnetic field. One now considers a uniform magnetic field $\mathbf{H}_{0}$ acting in the $x$-direction, and imagines a weak steady motion to take place in this field. The fluid is assumed to be homogeneous. The perturbation magnetic field will be denoted by $h$, so that the total magnetic field is

$$
\mathbf{H}=\mathbf{H}_{\mathbf{0}}+\mathbf{h} \text {. }
$$

The vector equation of motion is (all electromagnetic variables being measured in emu), with $\mathbf{g}$ as the body force per unit mass,

$$
\rho \frac{D \mathbf{v}}{D t}=-\operatorname{grad} p+\rho \mathbf{g}+\mu \mathbf{j} \times \mathbf{H}
$$

in which $\mathbf{v}$ is the velocity vector, $\mu$ is the magnetic permeability and $\mathbf{j}$ the current density. Since

$$
\operatorname{curl} \mathbf{H}=4 \pi \mathbf{j}
$$

Eq. (23) may be written

$$
\rho \frac{D \mathbf{v}}{D t}=-\operatorname{grad} p^{\prime}-\operatorname{grad}\left(\frac{\mu H^{2}}{8 \pi}\right)+\operatorname{div}\left(\frac{\mu \mathbf{H H}}{4 \pi}\right)
$$

in which

$$
p^{\prime}=p+\rho \phi
$$

with $\phi$ again denoting the potential of the body force. The intensity $\mathbf{E}$ of the electric field is related to the magnetic field by

$$
\operatorname{curl} \mathbf{E}=-\mu \frac{\partial \mathbf{H}}{\partial t}
$$

Furthermore, the current density, electric field, magnetic field, and velocity field are related by the equation

$$
\mathbf{j}=\sigma(\mathbf{E}+\mu \nabla \times \mathbf{H})
$$

in which $\sigma$ is the electric conductivity. The fluid being homogencous, the equation of continuity is again given by Eq. (5).

Since the motion under consideration is weak and steady, Eq. (25) becomes, after linearization:

$$
\begin{aligned}
& \frac{\partial p^{\prime}}{\partial x}=0, \\
& \frac{\partial p^{\prime}}{\partial y}=\frac{H_{0}}{4 \pi}\left(\frac{\partial h_{y}}{\partial x}-\frac{\partial h_{x}}{\partial y}\right), \\
& \frac{\partial p^{\prime}}{\partial z}=\frac{H_{0}}{4 \pi}\left(\frac{\partial h_{z}}{\partial x}-\frac{\partial h_{x}}{\partial z}\right) .
\end{aligned}
$$

From Eqs. (29) to (31) one obtains by cross differentiation

$$
\frac{\partial}{\partial x} \operatorname{curl} \mathbf{h}=0
$$


or, by virtue of Eq. (24),

$$
\frac{\partial}{\partial x} \mathbf{j}=\mathbf{0}
$$

so that the current density is independent of $x$. Although Eq. (33) is not needed to prove the independence of the fluid flow on $x$, it contains a result which is worthy of note.

The linearized form of Eq. (28) is

$$
\begin{aligned}
& j_{x}=\sigma E_{x}, \\
& j_{y}=\sigma\left(E_{y}+\mu H_{0} w\right), \\
& j_{x}=\sigma\left(E_{z}-\mu H_{0} v\right) .
\end{aligned}
$$

Since the motion under consideration is steady, Eq. (27) becomes

$$
\operatorname{curl} \mathbf{E}=\mathbf{0} \text {. }
$$

From Eqs. (24) and (34) to (37) one obtains, by the aid of Eq. (5),

$$
\frac{\partial}{\partial x} \nabla=\frac{\eta}{H_{0}} \nabla^{2} \mathbf{h}
$$

in which

$$
\eta=(4 \pi \mu \sigma)^{-1}
$$

is the magnetic viscosity. If the magnetic viscosity is negligible, Eq. (38) becomes

$$
\frac{\partial \mathbf{v}}{\partial x}=0
$$

so that the motion does not change with $x$. Thus the magnetic field seems to have endowed the fluid with a certain rigidity, as if the material along the lines of forces had been stiffened to give the fluid a fibrous structure. If the motion is axisymmetric (e.g., if a sphere is towed through the fluid), from the equation of continuity one can easily show that the radial velocity is zero. The motion is then one dimensional in any meridianal plane or, if no revolving motion exists, even truly one-dimensional. It may be mentioned that for small values of the magnetic viscosity the lines of force always move with the fluid, or, to use Alfven's expression, are "frozen" into the material (Cowling [6], pp. $5-6)$. In the case of steady weak motions, the word "frozen" used by Alfven acquires a much stronger meaning, as explained above.

5. Effect of an electric current. Consider now a uniform electric current $\mathbf{j}_{\mathbf{0}}$ flowing in the $z$-direction, with $(r, \theta, z)$ as the cylindrical coordinates. From Eq. (24) one obtains

$$
H_{\theta}=4 \pi j_{0} r
$$

the other two components of the main magnetic field being zero. Eliminating $\mathbf{j}$ and $\mathbf{E}$ from Eqs. (24), (27), and (28), one obtains

$$
\frac{\partial \mathbf{H}}{\partial t}=\operatorname{curl}(\mathbf{\nabla} \times \mathbf{H})+\eta \nabla^{2} \mathbf{H}
$$

which, for steady motion and negligible magnetic viscosity, becomes

$$
\operatorname{curl}(\boldsymbol{\nabla} \times \mathbf{H})=0 .
$$


To the first order, one has, with $(u, v, w)$ for $\left(v_{r}, v_{\theta}, v_{z}\right)$,

$$
\mathbf{\nabla} \times \mathbf{H}=\left(-H_{\theta} w, 0, H_{\theta} u\right)
$$

and

$$
\operatorname{curl}(\mathbf{\nabla} \times \mathbf{H})=\left\{\frac{1}{r} \frac{\partial}{\partial \theta}\left(H_{\theta} u\right),-\left[\frac{\partial}{\partial r}\left(H_{\theta} u\right)+\frac{\partial}{\partial z}\left(H_{0} w\right)\right], \frac{1}{r} \frac{\partial}{\partial \theta}\left(H_{0} w\right)\right\} .
$$

From Eqs. (41), (43) and (44) it follows that

$$
\begin{aligned}
\frac{\partial u}{\partial \theta} & =0 \\
\frac{\partial(r u)}{\partial r}+\frac{\partial(r w)}{\partial z} & =0 \\
\frac{\partial w}{\partial \theta} & =0 .
\end{aligned}
$$

But the equation of continuity is

$$
\frac{\partial(r u)}{\partial r}+\frac{\partial v}{\partial \theta}+\frac{\partial(r w)}{\partial z}=0
$$

Thus Eq. (46) yields

$$
\frac{\partial v}{\partial \theta}=0
$$

which, together with Eqs. (45) and (47), states that the motion is independent of $\theta$. The meaning of this statement can be fully realized physically by imagining an object to be towed slowly and steadily along a circular path about an axis parallel to the main electric current. According to the result just obtained, the fluid will be pushed along circular paths or along the lines of force of the main magnetic field induced by the main electric current. What has been said in the previous section about the "stiffening" of material along lines of force can be said in the present case also.

6. Concluding remarks. From the foregoing it may be concluded that, under the assumptions stated, the effects of rotation, gravity, or an electromagnetic field are to stiffen the fluid along the vorticity lines, isopycnic surfaces or lines, or lines of force, respectively. In the case of weak steady motions or relative motions, this "stiffening" has the effect of reducing the a priori number of physical or at least the mathematical dimensions of motion by one, in the various senses stated. In steady weak motions of a stratified fluid the velocity in the direction of gravity vanishes, and simple cases exist in which motion normal to vorticity lines or lines of forces are completely inhibited. For the motion of a conductive fluid due to the disturbance of an object slowly and steadily towed through it along a circular path, a torus-shaped mass of fluid will revolve around the axis of symmetry if a main uniform electric current is flowing longitudinally.

As already mentioned, the concept of a virtual gravitational field created by rotation helps to unify the understanding of many phenomena of fluid motion involving gravity and rotation.

7. Acknowledgment. This work is sponsored by the Office of Ordnance Research, U. S. Army. 


\title{
BIBLIOGIRAPIIY
}

1. J. Proudman, On the motion of solids in a liquid possessing vorticity, Proc. Roy. Soc. A92, 408-24 (1916)

2. Sir Geoffrey Taylor, Experiments on the motion of solid bodies in rotating fluids, Proc. Roy. Soc. A104, 213-18 (1923)

3. R. R. Long, Steady motion around a symmetrical obstacle moving along the axis of a rotating fluid, J. Met. 10, 197-203 (1953)

4. G. W. Morgan, A study of motions in a rotating liquid, Proc. Roy. Soc. A206, 108-30 (1951)

5. Paul Gariel, Recherches experimentales sur l'écoulement de couches superposées de fluides de densites differentes, La Houille Blanche, No. 1, 56-64 (1949)

6. T. G. Cowling, Magnetohydrodynamics, Interscience, 1957

\section{A GENERALIZATION OF LATTA'S METHOD FOR THE SOLUTION OF INTEGRAL EQUATIONS*}

\author{
BY MARVIN SHINBROT (Lockheed Aircraft Corporation)
}

1. Introduction. The integral equation

$$
\varphi(x)=\int_{a}^{b} k(x-t) f(t) d t, \quad a<x<b
$$

with $a$ and $b$ finite arises in many applications. As examples, we mention the problem of optimization of filters having a finite memory [1] and the problem of diffraction through a slit $[2,3]$. In the first of these problems, the kernel represents the autocorrelation of the inputs to the filter; in the second, $k(x)=H_{0}^{(1)}(|x|)$, the first Hankel function.

Its importance notwithstanding, no general solution of (1.1) is known; with but two exceptions, only special methods exist for application to special kernels. The first exception is the method developed not long ago by Latta $[4,5]$. The second-which is now subsumed under Latta's method-applies to the conceptually trivial case when the kernel has a rational Fourier transform [1].

Latta's method can be applied in the circumstance that $k(x)$ satisfies a linear differential equation (of any order) with linear coefficients. This restriction is still very far from being moderate, of course, and since no general solution of (1.1) appears to be forthcoming, it appears worthwhile to see whether there are other integral equations having the form of (1.1) which can be reduced to one of Latta's type.

In a recent paper [6], Pearson considered (1.1) with

$$
k(x)=p(x) \log |x|+q(x),
$$

where $p$ and $q$ were polynomials. Although l'earson's method has no apparent connection with Iatta's, the fact that $\log |x|$ is a Latta kernel leads one to consider kernels of the form

$$
. k(x)=p(x) j(x)+q(x),
$$

where $j$ satisfies a differential equation while, as in [6], $p$ and $q$ are polynomials. It is to consideration of this case that the present paper is devoted.

*Received October 23, 1957. This paper presents work done while the author was employed by the Nutional Advisory Committee for Aeronautics. 